\title{
Ideal magnetohydrodynamic stability of configurations without nested flux surfaces
}

\author{
P. Helander ${ }^{1}$ and S.L. Newton ${ }^{2}$ \\ ${ }^{1}$ Max-Planck-Institut für Plasmaphysik, EURATOM Association, 17491 Greifswald, \\ Germany \\ ${ }^{2}$ EURATOM/CCFE Association, Culham Science Centre, Abingdon, Oxon, OX14 \\ 3DB, United Kingdom
}

Existing numerical tools for calculating the MHD stability of magnetically confined plasmas generally assume the existence of nested flux surfaces. These tools are therefore not immediately applicable to configurations with magnetic islands or regions with an ergodic magnetic field. However, in practice these islands or ergodic regions are often small, and their effect on MHD stability can then be evaluated using a perturbation theory developed in the present paper. This procedure allows the effect of the broken magnetic topology on the stability of each eigenmode to be calculated without requiring any knowledge about the perturbed eigenfunctions. 


\section{Introduction}

The most commonly used numerical codes for calculating the equilibrium and stability of magnetically confined plasmas presume that the magnetic field possesses nested flux surfaces. In the case of stellarators, for example, the equilibrium is usually calculated using the VMEC [1] code, and its stability is assessed by codes such as CAS3D [2] or TERPSICHORE [3]. There are numerical tools capable of solving the force balance equation $\mathbf{J} \times \mathbf{B}=\nabla p$ in three dimensions without requiring nested flux surfaces (the PIES [4], HINT [5], SIESTA [6] and SPEC [7] codes), but there is at the moment no way of directly calculating the stability of the resulting equilibria. In tokamaks, axisymmetric equilibria are obtained from the Grad-Shafranov equation, and their stability is routinely evaluated by a large number of codes, but none of these is applicable if the magnetic topology is broken by error fields or intentionally produced resonant magnetic perturbations creating magnetic islands or regions with an ergodic magnetic field.

However, in practice configurations with broken flux surfaces are obviously useful only if the islands and ergodic regions are small. Any stellarator optimization attempts to maximize the regions of good flux surfaces [8], and in tokamaks the violation of axisymmetry is invariably a minor perturbation. The ideal MHD stability properties are then approximately the same as in an "unperturbed" equilibrium with nested flux surfaces $^{1}$, and the effect of the perturbation breaking these surfaces can be calculated by perturbation theory. It is the aim of the present work to show how this can be done, using no information other than that already available from existing numerical codes.

Before proceeding with the analyis, we emphasize that we do not consider nonlinear stability issues. Garabedian [11] and Cooper et al. [12] have shown that twodimensional equilbria can bifurcate into three-dimensional ones resembling the nonlinear state of a saturated kink mode. Nor do we consider resisitive stability, which can

\footnotetext{
${ }^{1} \mathrm{~A}$ small perturbation of an equilibrium can, in principle, also have a large effect on MHD stability. This is, for instance, the case if the equilibrium lies close to a stability boundary. More interestingly, a small resonant perturbation can cause a skin current on the corresponding magnetic surface, which could affect the MHD stability substantially. Such skin currents are difficult to resolve numerically, using ordinary equilibrium codes, but can be captured by using a perturbed-equilibrium approach $[9,10]$
} 
be calculated, both linearly and nonlinearly, using initial-value codes such as NIMROD [13] or M3D [14].

\section{Perturbation theory}

We consider the equilibrium of an ideal MHD plasma at rest with pressure $p(\mathbf{r})$ and density $\rho(\mathbf{r})$. The magnetic field $\mathbf{B}(\mathbf{r})$ that confines the plasma is assumed to possess toroidal nested flux surfaces, so that the stability can be calculated straightforwardly [15]. We then know the spectrum of eigenmodes $\boldsymbol{\xi}_{j}$ and eigenvalues $\omega_{j}^{2}$ satisfying the linearized force balance equation

$$
-\rho \omega_{j}^{2} \boldsymbol{\xi}_{j}=\mathbf{F}\left[\boldsymbol{\xi}_{j}\right]
$$

where $\mathbf{F}$ is the force density

$$
\mathbf{F}[\boldsymbol{\xi}]=-\nabla P+\mu_{0}^{-1}[(\nabla \times \mathbf{Q}) \times \mathbf{B}+(\nabla \times \mathbf{B}) \times \mathbf{Q}],
$$

with $\boldsymbol{\xi}$ the plasma displacement and

$$
\begin{gathered}
P=-\boldsymbol{\xi} \cdot \nabla p-\gamma p \nabla \cdot \boldsymbol{\xi}, \\
\mathbf{Q}=\nabla \times(\boldsymbol{\xi} \times \mathbf{B}),
\end{gathered}
$$

denoting the perturbations of the pressure and the magnetic field, respectively. (Note that $p$ denotes the pressure, whilst $P[\boldsymbol{\xi}]$ is a functional equal to the pressure perturbation.) We further consider a second equilibrium $(p+\delta p, \rho+\delta \rho, \mathbf{B}+\delta \mathbf{B})$ that deviates only a little from the first one but where the magnetic topology may be broken, so that magnetic islands or regions with chaotic field lines exist. The reason that the two equilibria deviate from one another will be different in different applications. In the case of stellarators, the first equilibrium may have been obtained from the VMEC code and the second equilibrium from PIES, HINT, SIESTA or SPEC. The first equilibrium may then have singular currents on rational flux surfaces that prevent magnetic islands from forming, whilst the second equilibrium will have islands around these surfaces. In tokamak applications, the first equilibrium may be axisymmetric and the second one could have had its symmetry perturbed by error fields or externally applied magnetic perturbations. Mathematically, the difference between the two equilibria then arises 
from different boundary conditions on the ideally conducting wall that we assume surrounds the plasma.

We begin by considering the case where the boundary conditions are the same for the two equilbria, and henceforth we shall refer to the second equilbrium as the "perturbed" one. Because of the broken flux surfaces, we are not immediately in a position to calculate the eigenfunctions and eigenvalues of the perturbed equilibrium, which are determined by the equation

$$
-(\rho+\delta \rho)\left(\omega_{j}^{2}+\delta \omega_{j}^{2}\right)\left(\boldsymbol{\xi}_{j}+\delta \boldsymbol{\xi}_{j}\right)=(\mathbf{F}+\delta \mathbf{F})\left[\boldsymbol{\xi}_{j}+\delta \boldsymbol{\xi}\right]
$$

where $\mathbf{F}+\delta \mathbf{F}$ is the perturbed force operator,

$\mathbf{F}+\delta \mathbf{F}=-\nabla(P+\delta P)+\mu_{0}^{-1}[(\nabla \times(\mathbf{Q}+\delta \mathbf{Q})) \times(\mathbf{B}+\delta \mathbf{B})+(\nabla \times(\mathbf{B}+\delta \mathbf{B}) \times(\mathbf{Q}+\delta \mathbf{Q})]$, and for an arbitrary vector field $\boldsymbol{\eta}$

$$
\begin{gathered}
\delta P[\boldsymbol{\eta}]=-\boldsymbol{\eta} \cdot \nabla \delta p-\gamma \delta p \nabla \cdot \boldsymbol{\eta}, \\
\delta \mathbf{Q}[\boldsymbol{\eta}]=\nabla \times(\boldsymbol{\eta} \times \delta \mathbf{B}) .
\end{gathered}
$$

(Again, note that $\delta p$ denotes the change in the equilibrium pressure whereas $\delta P[\boldsymbol{\eta}]$ is a functional of $\boldsymbol{\eta}$.) However, an expression for the change, $\delta \omega_{j}^{2}$, in the eigenvalue $\omega_{j}^{2}$ can be derived in a way familiar from perturbation theory in quantum mechanics, by multiplying the complex conjugate of Eq. (1) by $\delta \boldsymbol{\xi}_{j}$ and integrating over the plasma volume $V$,

$$
-\int_{V} \rho \omega_{j}^{2} \delta \boldsymbol{\xi}_{j} \cdot \boldsymbol{\xi}_{j}^{*} d V=\int_{V} \delta \boldsymbol{\xi}_{j} \cdot \mathbf{F}\left[\boldsymbol{\xi}_{j}^{*}\right] d V
$$

Next, we subtract Eq. (1) from Eq. (2), neglect terms that are quadratic in the perturbations, multiply by $\boldsymbol{\xi}_{j}^{*}$ and integrate over $V$,

$$
-\int_{V}\left[\rho \omega_{j}^{2} \boldsymbol{\xi}_{j}^{*} \cdot \delta \boldsymbol{\xi}_{j}+\left(\rho \delta \omega_{j}^{2}+\omega_{j}^{2} \delta \rho\right)\left|\boldsymbol{\xi}_{j}\right|^{2}\right] d V=\int_{V} \boldsymbol{\xi}_{j}^{*} \cdot\left(\mathbf{F}\left[\boldsymbol{\xi}_{j}\right]+\delta \mathbf{F}\left[\boldsymbol{\xi}_{j}\right]\right) d V .
$$

Subtracting Eq. (3) from this result gives

$$
-\int_{V}\left(\rho \delta \omega_{j}^{2}+\omega_{j}^{2} \delta \rho\right)\left|\boldsymbol{\xi}_{j}\right|^{2} d V=\int_{V} \boldsymbol{\xi}_{j}^{*} \cdot \delta \mathbf{F}\left[\boldsymbol{\xi}_{j}\right] d V+\int_{V}\left(\boldsymbol{\xi}_{j}^{*} \cdot \mathbf{F}\left[\delta \boldsymbol{\xi}_{j}\right]-\delta \boldsymbol{\xi}_{j} \cdot \mathbf{F}\left[\boldsymbol{\xi}_{j}^{*}\right]\right) d V,
$$

where the last term vanishes thanks to the self-adjointness of $\mathbf{F}$ [15]. We thus obtain our main result

$$
\delta \omega_{j}^{2}=-\int_{V}\left(\boldsymbol{\xi}_{j}^{*} \cdot \delta \mathbf{F}\left[\boldsymbol{\xi}_{j}\right]-\omega_{j}^{2}\left|\boldsymbol{\xi}_{j}\right|^{2} \delta \rho\right) d V / \int_{V} \rho\left|\boldsymbol{\xi}_{j}\right|^{2} d V
$$


which makes it possible to determine how much more or less stable each eigenmode becomes as a result of perturbing the equilibrium. It is important to note that no knowledge is required about the perturbed eigenfunctions. Only the unperturbed eigenfunctions and the perturbed equilibrium are needed to calculate $\delta \omega_{j}^{2}$. If this quantity is positive, the perturbed equilibrium is more stable than the unperturbed one to the eigenmode in question, and if $\delta \omega_{j}^{2}$ is negative it is less stable.

Note that a perturbation in the density profile alone, without changing the pressure or the magnetic field, causes $\delta \rho \neq 0$ and $\delta \mathbf{F}=0$, and therefore cannot make an unstable mode stable, or vice versa, according to Eq. (6).

\subsection{Validity}

In deriving Eq. (6), we have neglected terms that are quadratic in the perturbations, which are assumed to be small, but the treatment nevertheless does not exclude the possiblility that a mode may cross the stability boundary as a result of the perturbation, although this requires $\delta \omega_{j}^{2}>\omega_{j}^{2}$. To see why, let us now retain all terms containing $\delta \omega_{j}^{2}$, including those that involve perturbations squared. Instead of Eq. (4) we then obtain

$$
\begin{gathered}
-\int_{V}\left[\rho\left(\omega_{j}^{2}+\delta \omega_{j}^{2}\right) \boldsymbol{\xi}_{j}^{*} \cdot \delta \boldsymbol{\xi}_{j}+\left(\rho \delta \omega_{j}^{2}+\omega_{j}^{2} \delta \rho+\delta \rho \delta \omega_{j}^{2}\right)\left|\boldsymbol{\xi}_{j}\right|^{2}\right] d V \\
=\int_{V} \boldsymbol{\xi}_{j}^{*} \cdot\left(\mathbf{F}\left[\boldsymbol{\xi}_{j}\right]+\delta \mathbf{F}\left[\boldsymbol{\xi}_{j}\right]\right) d V
\end{gathered}
$$

and instead of Eq. (5)

$$
-\int_{V}\left[\left((\rho+\delta \rho) \delta \omega_{j}^{2}+\omega_{j}^{2} \delta \rho\right)\left|\boldsymbol{\xi}_{j}\right|^{2}+\rho \delta \omega_{j}^{2} \boldsymbol{\xi}_{j}^{*} \cdot \delta \boldsymbol{\xi}_{j}\right] d V=\int_{V} \boldsymbol{\xi}_{j}^{*} \cdot \delta \mathbf{F}\left[\boldsymbol{\xi}_{j}\right] d V
$$

whence it is clear that Eq. (6) holds even if $\delta \omega_{j}^{2}$ is comparable to $\omega_{j}^{2}$, because $\delta \rho \ll \rho$ and

$$
\int_{V} \rho \boldsymbol{\xi}_{j}^{*} \cdot \delta \boldsymbol{\xi}_{j} d V \ll \int_{V} \rho\left|\boldsymbol{\xi}_{j}\right|^{2} d V
$$

Equation (6) is also valid if the unperturbed equilibrium $(p, \rho, \mathbf{B})$ is only approximately correct. For instance, if the true equilibrium is given by $(p+\delta p, \rho+\delta \rho, \mathbf{B}+\delta \mathbf{B})$ and contains small magnetic islands, but can be approximated by an island-free but approximate equilibrium $(p, \rho, \mathbf{B})$ whose eigenfunctions $\boldsymbol{\xi}_{j}$ and eigenvalues $\omega_{j}^{2}$ are known, then the eigenvalues $\omega_{j}^{2}+\delta \omega_{j}^{2}$ of the true equilibrium can be obtained from Eq. (6). This is potentially very useful, because it allows the MHD stability properties of an 
equilibrium with small islands to be calculated even if there is no exact nearby equilibrium without islands. It is perhaps not immediately obvious that this should be possible, because if $(p, \rho, \mathbf{B})$ is only approximately an equilibrium then the operator $\mathbf{F}$ is not exactly self-adjoint and the last integral in Eq. (5) does not vanish. Nevertheless, if the equilibrium $(p+\delta p, \rho+\delta \rho, \mathbf{B}+\delta \mathbf{B})$ is exact then $\mathbf{F}+\delta \mathbf{F}$ is self-adjoint and $\mathbf{F}$ is nearly so, making the last term in Eq. (5) second order.

\subsection{Perturbation of the boundary}

The result (6) is however not valid if the boundary is perturbed. This boundary is assumed to be stationary and ideally conducting, but in many cases of interest the two equilibria $(p, \rho, \mathbf{B})$ and $(p+\delta p, \rho+\delta \rho, \mathbf{B}+\delta \mathbf{B})$ occupy slightly different regions, $V$ and $W$, say. For instance, the boundary of an axisymmetric plasma may be perturbed in such a way that resonant magnetic perturbations are created and magnetic islands inside arise. In such cases the right-hand side of Eq. (6) must be supplemented by a term describing the boundary perturbation.

The simplest situation occurs if the ideally conducting wall is immediately adjacent to the plasma, without a vacuum region separating the plasma from the wall. The boundary condition on the displacement $\boldsymbol{\xi}$ is then $\xi_{n}=\boldsymbol{\xi} \cdot \hat{\mathbf{n}}=0$, where $\hat{\mathbf{n}}$ is the unit normal vector, since the normal component of the plasma velocity $\mathbf{v}=-i \omega \boldsymbol{\xi}$ then vanishes and the condition

$$
\hat{\mathbf{n}} \times(\mathbf{E}+\mathbf{v} \times \mathbf{B})=\hat{\mathbf{n}} \times \mathbf{E}+\hat{\mathbf{n}} \cdot \mathbf{B v}-\hat{\mathbf{n}} \cdot \mathbf{v B}=0,
$$

implies $\hat{\mathbf{n}} \times \mathbf{E}=0$, as required near a perfectly conducting wall. If we denote the boundaries of the two equilibria by $\partial V$ and $\partial W$, respectively, and the perpendicular distance between them by $\epsilon(\mathbf{r})$, then the normal displacement satisfies

$$
\begin{gathered}
\xi_{n}\left(\mathbf{r}_{0}\right)=0 \\
\xi_{n}\left(\mathbf{r}_{0}+\epsilon \hat{\mathbf{n}}\right)+\delta \xi_{n}\left(\mathbf{r}_{0}+\epsilon \hat{\mathbf{n}}\right)=0
\end{gathered}
$$

if $\mathbf{r}_{0} \in \partial V$. Here $\xi_{n}=\hat{\mathbf{n}} \cdot \boldsymbol{\xi}_{j}$ and $\xi_{n}+\delta \xi_{n}=\hat{\mathbf{n}} \cdot\left(\boldsymbol{\xi}_{j}+\delta \boldsymbol{\xi}_{j}\right)$ denote the normal components of the eigenvectors corresponding to the two equilibria. (The subscript $j$ will be suppressed.) If $V$ and $W$ almost coincide so that $\epsilon$ is small, the boundary condition on 
the perturbed eigenfunctions taken on the unperturbed boundary $\partial V$ is thus

$$
\delta \xi_{n}\left(\mathbf{r}_{0}\right)=-\epsilon \hat{\mathbf{n}} \cdot \nabla \xi_{n}
$$

Since this quantity does not vanish in general, the last integral in Eq. (5) does not either; the self-adjointness of $\mathbf{F}$ holds only for displacements without normal component on the boundary. The contribution from this integral is however easily evaluated from Eq. (C3) of Ref. [16],

$$
\begin{gathered}
\int_{V}(\boldsymbol{\eta} \cdot \mathbf{F}[\boldsymbol{\lambda}]-\boldsymbol{\lambda} \cdot \mathbf{F}[\boldsymbol{\eta}]) d V \\
=\int_{\partial V}\left\{\boldsymbol{\eta}\left[\gamma p \nabla \cdot \boldsymbol{\lambda}-\mu_{0}^{-1}(\mathbf{Q}(\boldsymbol{\lambda}) \cdot \mathbf{B}+\boldsymbol{\lambda} \cdot \nabla \mathbf{B} \cdot \mathbf{B})+\boldsymbol{\lambda} \cdot \nabla\left(p+\frac{B^{2}}{2 \mu_{0}}\right)\right]\right. \\
\left.-\boldsymbol{\lambda}\left[\gamma p \nabla \cdot \boldsymbol{\eta}-\mu_{0}^{-1}(\mathbf{Q}(\boldsymbol{\eta}) \cdot \mathbf{B}+\boldsymbol{\eta} \cdot \nabla \mathbf{B} \cdot \mathbf{B})+\boldsymbol{\eta} \cdot \nabla\left(p+\frac{B^{2}}{2 \mu_{0}}\right)\right]\right\} \cdot \hat{\mathbf{n}} d S,
\end{gathered}
$$

where $\boldsymbol{\lambda} \cdot \nabla \mathbf{B} \cdot \mathbf{B}=\boldsymbol{\lambda} \cdot \nabla\left(B^{2} / 2\right)$. Choosing $\boldsymbol{\lambda}=\boldsymbol{\xi}_{j}^{*}$ and $\boldsymbol{\eta}=\delta \boldsymbol{\xi}_{j}$ gives

$$
\int_{V}\left(\boldsymbol{\xi}_{j}^{*} \cdot \mathbf{F}\left[\delta \boldsymbol{\xi}_{j}\right]-\delta \boldsymbol{\xi}_{j} \cdot \mathbf{F}\left[\boldsymbol{\xi}_{j}^{*}\right]\right) d V=-\int_{\partial V}\left[\gamma p \nabla \cdot \boldsymbol{\xi}_{j}^{*}-\left(\nabla \times\left(\boldsymbol{\xi}_{j}^{*} \times \mathbf{B}\right)\right) \cdot \mathbf{B} / \mu_{0}\right] \hat{\mathbf{n}} \cdot \nabla\left(\hat{\mathbf{n}} \cdot \boldsymbol{\xi}_{j}\right) \epsilon d S,
$$

which should be added to the numerator of Eq. (6) if the boundary is perturbed.

If both the normal component of the eigenfunction $\hat{\mathbf{n}} \cdot \boldsymbol{\xi}_{j}$ and its normal derivative vanishes on the boundary $\partial V$, then Eq. (6) is valid without correction. This is, for instance, the case for ordinary ballooning modes whose eigenfunctions are localized to the plasma interior.

\subsection{Reformulation}

The contribution of $\delta F$ to (6) may be rewritten in a form similar to that appearing in the standard form of the fluid contribution to the energy principle (see for example Eq. (8.75) of Ref. [15]). In the usual energy principle, the reformulation is done by using the identity obtained in proving the self-adjointness of $\mathbf{F}$, Eq. (B1) of Ref. [16] or Eq. (A4) of Ref. [15],

$$
\mathbf{B} \cdot[\nabla(\boldsymbol{\xi} \cdot \nabla p)+\mathbf{J} \times \mathbf{Q}]=0,
$$

where $\mathbf{J}=\mu_{0}^{-1} \nabla \times \mathbf{B}$ is the equilibrium current density. Therefore, denoting the perturbed current density $\delta \mathbf{J}=\mu_{0}^{-1} \nabla \times \delta \mathbf{B}$, we consider the parallel projection

$$
\mathbf{B} \cdot[\nabla(\boldsymbol{\xi} \cdot \nabla \delta p)+\delta \mathbf{J} \times \mathbf{Q}+\mathbf{J} \times \delta \mathbf{Q}],
$$


taking parallel and perpendicular with respect to the initial equilibrium, to determine a similar identity for the perturbed force operator.

Remembering that the equilibrium satisfies $\mathbf{J} \times \mathbf{B}=\nabla p$ and so $\delta \mathbf{J} \times \mathbf{B}+\mathbf{J} \times \delta \mathbf{B}=$ $\nabla \delta p$, using standard vector identities the above contributions are

$$
\begin{aligned}
\mathbf{B} \cdot[\nabla(\boldsymbol{\xi} \cdot \nabla \delta p)] & =\nabla \cdot[(\boldsymbol{\xi} \cdot \nabla \delta p) \mathbf{B}], \\
\mathbf{B} \cdot(\mathbf{J} \times \delta \mathbf{Q}) & =-\nabla p \cdot \delta \mathbf{Q} \\
& =\nabla \cdot(\nabla p \times(\boldsymbol{\xi} \times \delta \mathbf{B})) \\
& =\nabla \cdot[(\delta \mathbf{B} \cdot \nabla p) \boldsymbol{\xi}-(\boldsymbol{\xi} \cdot \nabla p) \delta \mathbf{B}], \\
\mathbf{B} \cdot(\delta \mathbf{J} \times \mathbf{Q}) & =-(\nabla \delta p-\mathbf{J} \times \delta \mathbf{B}) \cdot \mathbf{Q} \\
& =\nabla \cdot(\nabla \delta p \times(\boldsymbol{\xi} \times \mathbf{B}))+\mathbf{Q} \cdot \mathbf{J} \times \delta \mathbf{B} \\
& =\nabla \cdot[(\mathbf{B} \cdot \nabla \delta p) \boldsymbol{\xi}-(\boldsymbol{\xi} \cdot \nabla \delta p) \mathbf{B}]+\mathbf{Q} \cdot \mathbf{J} \times \delta \mathbf{B} .
\end{aligned}
$$

Noting from the perturbed equilibrium condition that $\mathbf{B} \cdot \nabla \delta p=-\delta \mathbf{B} \cdot \mathbf{J} \times \mathbf{B}$, the total projection can be reduced to

$$
\mathbf{B} \cdot[\nabla(\boldsymbol{\xi} \cdot \nabla \delta p)+\delta \mathbf{J} \times \mathbf{Q}+\mathbf{J} \times \delta \mathbf{Q}]=-\delta \mathbf{B}_{\perp} \cdot\left[\nabla\left(\boldsymbol{\xi}_{\perp} \cdot \nabla p\right)+\mathbf{J} \times \mathbf{Q}\right],
$$

noting that $\boldsymbol{\xi}_{\|} \cdot \nabla p=0$ and from the usual identity Eq. (8), that the parallel component of the bracket on the right of Eq. (9) is zero.

As in the standard formulation, we can write the perturbed eigenfunction as components $\boldsymbol{\xi}=\boldsymbol{\xi}_{\perp}+\xi_{\|} \hat{\mathbf{b}}$, where $\hat{\mathbf{b}}=\mathbf{B} /|\mathbf{B}|$ is a unit vector in the direction of the equilibrium magnetic field. The contribution of $\delta \mathbf{F}$ to the integral in Eq. (6) is then

$$
\begin{aligned}
\boldsymbol{\xi}^{*} \cdot \delta \mathbf{F}[\boldsymbol{\xi}]=\mu_{0}^{-1} \boldsymbol{\xi}^{*} \cdot[(\nabla & \times \delta \mathbf{Q}) \times \mathbf{B}+(\nabla \times \mathbf{Q}) \times \delta \mathbf{B}]+\gamma \boldsymbol{\xi}^{*} \cdot \nabla(\delta p \nabla \cdot \boldsymbol{\xi}) \\
& +\boldsymbol{\xi}^{*} \cdot[\nabla(\boldsymbol{\xi} \cdot \nabla \delta p)+\delta \mathbf{J} \times \mathbf{Q}+\mathbf{J} \times \delta \mathbf{Q}] \\
=\mu_{0}^{-1} \boldsymbol{\xi}^{*} \cdot[(\nabla & \times \delta \mathbf{Q}) \times \mathbf{B}+(\nabla \times \mathbf{Q}) \times \delta \mathbf{B}]+\gamma \boldsymbol{\xi}^{*} \cdot \nabla(\delta p \nabla \cdot \boldsymbol{\xi}) \\
& +\boldsymbol{\xi}_{\perp}^{*} \cdot[\nabla(\boldsymbol{\xi} \cdot \nabla \delta p)+\delta \mathbf{J} \times \mathbf{Q}+\mathbf{J} \times \delta \mathbf{Q}] \\
& -\frac{1}{B} \xi_{\|}^{*} \delta \mathbf{B}_{\perp} \cdot\left[\nabla\left(\boldsymbol{\xi}_{\perp} \cdot \nabla p\right)+\mathbf{J} \times \mathbf{Q}\right] .
\end{aligned}
$$

In the case where the boundary is unperturbed, this expression may be conveniently integrated by parts and its contribution to Eq. (6) finally reduced to

$$
-\int_{V} \boldsymbol{\xi}^{*} \cdot \delta \mathbf{F}[\boldsymbol{\xi}] d V=\frac{1}{\mu_{0}} \int_{V}\left(\delta \mathbf{Q} \cdot \mathbf{Q}^{*}+\mathbf{Q} \cdot \delta \mathbf{Q}^{*}\right) d V+\gamma \int_{V} \delta p|\nabla \cdot \boldsymbol{\xi}|^{2} d V
$$




$$
\begin{aligned}
& +\int_{V}\left[\left(\boldsymbol{\xi}_{\perp} \cdot \nabla \delta p\right)\left(\nabla \cdot \boldsymbol{\xi}_{\perp}^{*}\right)-\boldsymbol{\xi}_{\perp}^{*} \cdot(\delta \mathbf{J} \times \mathbf{Q}+\mathbf{J} \times \delta \mathbf{Q})\right] d V \\
& +\int_{V}\left[\left(\xi_{\|} \hat{\mathbf{b}} \cdot \nabla \delta p\right)\left(\nabla \cdot \boldsymbol{\xi}_{\perp}^{*}\right)+\frac{1}{B} \xi_{\|}^{*} \delta \mathbf{B}_{\perp} \cdot\left(\nabla\left(\boldsymbol{\xi}_{\perp} \cdot \nabla p\right)+\mathbf{J} \times \mathbf{Q}\right)\right] d V
\end{aligned}
$$

\subsection{Application to tokamaks}

If the unperturbed equilibrium is axisymmetric, it is useful to Fourier decompose the eigenfunctions

$$
\boldsymbol{\xi}_{j}(r, \theta, \varphi)=\hat{\boldsymbol{\xi}}_{j}(r, \theta) e^{i n \varphi}
$$

where $r$ denotes a flux-surface label, and $(\theta, \varphi)$ the poloidal and toroidal angles. It is then clear from Eq. (6) that only the axisymmetric components of $\delta \mathbf{F}$ and $\delta \rho$ will contribute to

$$
\delta \omega_{j}^{2}=-\int_{V}\left(\hat{\boldsymbol{\xi}}_{j}^{*} e^{-i n \varphi} \cdot \delta \mathbf{F}\left[\hat{\boldsymbol{\xi}}_{j} e^{i n \varphi}\right]-\omega_{j}^{2}\left|\hat{\boldsymbol{\xi}}_{j}\right|^{2} \delta \rho\right) d V / \int_{V} \rho\left|\hat{\boldsymbol{\xi}}_{j}\right|^{2} d V
$$

Such components arise, for instance, because of the overall flattening of the pressure profile due to a chain of magnetic islands or ergodic field lines.

\section{Summary}

We have presented a procedure by which the ideal MHD stability of a magnetically confined plasma without nested flux surfaces may be determined, as long as any magnetic islands or ergodic regions are small. The result is expressed in Eqs. (6) and (7), which give the relative change in stability compared to that of a nearby equilibrium, for which the eigenfunctions are known. Crucially, knowledge of the eigenfunctions of the perturbed equilibrium is not required. In general, we envisage the result (6) to be evaluated numerically, using output from numerical codes. All that is needed, in principle, is to numerically evaluate volume integrals of known quantities, namely, the unperturbed eigenfunctions and the field perturbations.

\section{Acknowledgements}

Helpful discussions with Carolin Nührenberg and Joachim Geiger are gratefully acknowledged. This work was part-funded by the RCUK Energy Programme [grant number EP/I501045] and the European Communities under contracts of Association 
between EURATOM and CCFE/IPP. The views and opinions expressed herein do not necessarily reflect those of the European Commission.

\section{References}

[1] S.P. Hirshman, W.I. van Rij and P. Merkel, Comput. Phys. Comm. 43, 143 (1986).

[2] C. Nührenberg, Phys. Plasmas 6, 137 (1999).

[3] D.V. Anderson, W.A. Cooper, R. Gruber, S. Merazzi and U. Schwenn, Int. J. Supercomp. Appl. 4, 34 (1990).

[4] A.H. Reiman and H.S. Greenside, Comput. Phys. Commun. 43, 157 (1986).

[5] Y. Suzuki, N. Nakajima, K. Watanabe, Y. Nakamura and T. Hayashi, Nucl. Fusion 46, L19 (2006).

[6] S.P. Hirshman, R. Sanchez and C.R. Cook, Phys. Plasmas 18, 062504 (2011).

[7] S.R. Hudson, R.L. Dewar, G. Dennis, M.J. Hole, M. McGann et al., Phys. Plasmas 19, 112502 (2012).

[8] J.D. Hanson and J.R. Cary, Phys. Fluids 27, 767 (1984).

[9] J.-K. Park, A. H. Boozer, and A. H. Glasser, Phys. Plasmas 14, 052110 (2007).

[10] C. Nührenberg, A.H. Boozer, and S.R. Hudson, Phys. Rev. Lett. 102, 235001 (2009).

[11] P.R. Garabedian, Proc. Nat. Acad. Sci. 105, 13716 (2008).

[12] W.A. Cooper, J.P. Graves, A. Pochelon, O. Sauter, and L. Villard, Phys. Rev. Lett. 105, 035003 (2010).

[13] C.R. Sovinec, A.H. Glasser, D.C. Barnes, T.A. Gianakon, R.A. Nebel et al., J. Comp. Phys. 195, 355 (2004).

[14] J. Chen, J. Breslau, G. Fu, S. Jardin and W. Park, Comp. Phys. Comm. 164, 468 (2004).

[15] J.P. Freidberg, Ideal Magnetohydrodynamics (Plenum Press, 1987). 
[16] I.B. Bernstein, in Handbook of Plasma Physics, edited by M.N. Rosenbluth and R.Z. Sagdeev (North-Holland Publishing Company, 1983) Vol. 1, p. 421. 